\title{
1 Mindfulness-based therapy regulates brain connectivity in major depression
}

Michael Lifshitz ${ }^{1,2^{*}}$, Matthew Sacchet ${ }^{3 *}$, Julia M. Huntenburg ${ }^{4,5}$, Thomas Thiery ${ }^{6}$, Yan Fan ${ }^{7,8}$, Matti Gärtner $^{8}$, Simone Grimm ${ }^{8}$, Emilia Winnebeck ${ }^{8,9}$, Maria Fissler ${ }^{8,9}$, Titus A. Schroeter ${ }^{9}$, Daniel S. Margulies $^{4 \dagger}$, Thorsten Barnhofer ${ }^{10 \dagger}$

*Equal contribution

${ }^{\dagger}$ Equal contribution

${ }^{1}$ Integrated Program in Neuroscience, McGill University, Montreal, Québec, Canada

${ }^{2}$ Department of Anthropology, Stanford University, Stanford, California, USA

${ }^{3}$ Center for Depression, Anxiety, and Stress Research, McLean Hospital, Harvard Medical School, Belmont, Massachusets, USA

${ }^{4}$ Max Planck Research Group for Neuroanatomy \& Connectivity, Max Planck Institute for Human Cognitive and Brain Sciences, Leipzig, Germany

${ }^{5}$ Neurocomputation and Neuroimaging Unit, Department of Education and Psychology, Free University of Berlin, Berlin, Germany

${ }^{6}$ Department of Psychology, Université de Montréal, Montreal, Québec, Canada

${ }^{7}$ Department of Psychology and Neurosciences, Leibniz Research Centre for Working Environment and Human Factors, TU Dortmund University, Dortmund, Germany

${ }^{8}$ Clinic for Psychiatry and Psychotherapy, Charité University Medicine Berlin, Berlin, Germany

${ }^{9}$ Dahlem Institute for Neuroimaging of Emotion, Freie Universität, Berlin, Germany

${ }^{10}$ School of Psychology, University of Surrey, Guildford, UK

Short title: Mindfulness regulates brain connectivity in depression

Please address correspondence to:

Michael Lifshitz

Department of Anthropology

Stanford University, Building 50, 450 Serra Mall, Stanford, California, USA, 94305.

Email: lifshitz@stanford.edu

Phone: +1 (650) 723-3421

Keywords: major depression; mindfulness-based therapy; non-pharmacological intervention; functional connectivity; frontoparietal network. 


\section{Text}

Dear Editor,

Major depressive disorder (MDD) is associated with abnormal functional interactions among large-scale brain networks [1]. The development of more comprehensive neural models of MDD promises to inform treatment by targeting the modulation of specific brain circuits. Here we report findings from a randomized, active-controlled trial examining whether mindfulness-based therapy - a clinically effective non-pharmacological treatment for depression-can regulate specific patterns of functional brain connectivity in clinically depressed patients.

Mindfulness-based therapy is rapidly gaining popularity as an evidence-based treatment for depression [2]. What distinguishes mindfulness-based therapies from other psychological interventions is their emphasis on meditative training designed to promote attention, interoceptive awareness, and selfregulation. Prior research investigating healthy populations has demonstrated that meditation training can induce functional and structural plasticity within key nodes of the frontoparietal, default, and salience networks [3, 4]-brain circuits centrally implicated in the pathophysiology of MDD [1]. However, despite promising clinical data from well-controlled trials [2], the neural mechanisms of mindfulness in the treatment of depression remain unknown.

For the first time, this study used fMRI to examine the impact of mindfulness-based therapy on brain function in MDD. Specifically, we investigated the effects of a brief mindfulness-based intervention on resting-state functional connectivity in individuals with recurrent MDD. Patients were randomized to either a two-week mindfulness-based therapy (consisting of three individual face-to-face sessions and daily guided home practice) or a relaxation-based control intervention. The control condition mirrored the mindfulness intervention in terms of practice structure and time commitment, allowing us to specify the impact of meditative training beyond nonspecific factors such as provision of a rationale, therapist contact, and quiet rest. Before and after treatment, resting-state fMRI data were acquired. Data from thirty-one participants were suitable for analysis.

At the behavioural level, mindfulness-based therapy led to significant decreases in depressive symptoms (as measured by the Beck Depression Inventory-II) relative to the control intervention (Fig. 1C). In terms 
of brain changes, networks of interest were identified a priori based on the meditation and MDD neuroimaging literatures. Functional connectivity was quantified using a standard seed-based approach. We placed $10 \mathrm{~mm}$ seeds centered on each of bilateral dorsolateral prefrontal cortices (DLPFC), bilateral anterior insula (aINS), and bilateral posterior cingulate cortex (PCC) for the frontoparietal, salience, and default networks, respectively. Next, we implemented a spreading interaction approach (as in 5) to specifically identify voxels in which the mindfulness group exhibited change from pre- to post-treatment while the control group did not.

As displayed in Fig. 1A, whole-brain analyses yielded three statistically significant clusters related to the DLPFC seed: bilateral fusiform gyrus (right: 140 voxels, peak voxel MNI coordinates [24, -51, -12]; left: 69 voxels, [-24, -63, -15]) and right angular gyrus (248 voxels, [36, -78, 21]). The significant spreading interactions were driven by decreases in DLPFC connectivity from pre- to post-treatment in the mindfulness group while the control group signal did not change (Fig. 1B). Whole-brain analyses related to the aINS and PCC seeds did not yield statistically significant results. It is important to note that we had a small sample size and so our findings should be interpreted with due caution pending replication.

These results show that mindfulness-based therapy for MDD ameliorates clinical symptoms while regulating resting-state functional connectivity, over and above the effects of a relaxation-based control intervention. We found that two-weeks of mindfulness-based therapy reduced connectivity between the frontoparietal control network (DLPFC) and regions involved in higher-order processing of sensory input (bilateral fusiform gyrus and right angular gyrus, which spanned the visual, frontoparietal, and dorsalattention networks). Our results extend previous findings showing that psychological treatments for MDD can modulate functional connectivity in relevant brain networks [6]. However, whereas prior studies lacked a control treatment group, our study is the first active-controlled report to demonstrate that a psychological intervention exerts a specific influence on brain connectivity in MDD.

We found that mindfulness-based therapy reduced connectivity between the DLPFC seed and bilateral fusiform gyrus. As part of the ventral visual stream in the canonical visual network, the fusiform gyrus plays an important role in higher-order processing of incoming visual information, including social and emotional cues [7]. The present finding aligns with the results of a prior study of long-term meditators, which similarly showed decreased resting-state functional connectivity between the DLPFC and regions 
101 of the visual network (including cuneus and occipital gyrus) [8]. The fusiform gyrus in particular has

102 been implicated in studies of meditation [3, 4] as well as clinical depression and antidepressant drug 103 action [9].

105 The mindfulness-based intervention also reduced connectivity between the DLPFC seed and a cluster in

106 the right angular gyrus. This cluster was centered in the canonical visual network and spanned into the 107 frontoparietal and dorsal-attention networks. Meta-analytic findings link MDD to dampened connectivity 108 both within and between the frontoparietal and dorsal-attention networks [1]; thus, contrary to our 109 findings, we might have expected the mindfulness treatment to increase connectivity between the DLPFC 110 and this angular gyrus cluster. On the other hand, at least four studies have reported increased 111 connectivity between frontoparietal network regions in patients with MDD [1]. Moreover, an 112 investigation of successful electroconvulsive therapy for severe MDD revealed substantial decreases in 113 frontoparietal network connectivity [10]. That study was the only other investigation of MDD treatment, 114 besides the present report, to show changes in connectivity between regions of the frontoparietal network; 115 thus, it is noteworthy that connectivity of this network was reduced as a result of intervention, as is 116 consistent with our current findings.

118 In conclusion, the present report elucidates the impact of mindfulness-based therapy on functional brain 119 organization in major depression. We demonstrate, using a randomized active-controlled design, that a 120 brief, clinically effective mindfulness intervention functionally decouples top-down control regions from 121 brain areas implicated in sensory, affective, and attentional processing. While previous work has 122 demonstrated the clinical impact of mindfulness training, the present findings shed light on the precise 123 neural targets, providing new insight into the specificity of this therapeutic approach. 


\section{2. Statements}

125

126

\subsection{Acknowledgment}

127 We thank our study participants for their time and effort. In addition, we are grateful to Ishan Walpola 128 for helpful suggestions throughout the preparation of this manuscript. The study is registered at 129 ClinicalTrials.gov (NCT02801513).

130

\section{1}

\subsection{Disclosure Statement}

132 The authors have no conflicts of interest to declare.

133

\section{$134 \quad 2.3$ Statement of Ethics}

135 The study protocol was approved by the ethics committee of the Charité University Medicine Berlin, 136 Campus Mitte (EA4/037/11). All participants provided written informed consent.

\section{$138 \quad 2.4$ Funding Sources}

139 Michael Lifshitz acknowledges a Francisco J. Varela Research Award from the Mind and Life Institute 140 and a Vanier Graduate Scholarship from the Natural Sciences and Engineering Research Council of 141 Canada. Thorsten Barnhofer acknowledges support by a Heisenberg Fellowship from the German 142 Research Foundation (BA2255 2-1). This research was funded by German Research Foundation 143 (Deutsche Forschungsgemeinschaft) Grant BA2255 3-1, awarded to Thorsten Barnhofer. The funders

144 had no role in study design; in the collection, analysis and interpretation of data; in the writing of the 145 report; or in the decision to submit the article. 
Lifshitz et al. 6

146

147

148

149

150

151

152

153

154

155

156

157

158

159

160

161

162

163

164

165

166

167

168

169

170

171

172

173

174

175

176

177

\section{References}

1. Kaiser RH, Andrews-Hanna JR, Wager TD, Pizzagalli DA. Large-scale network dysfunction in major depressive disorder: A meta-analysis of resting-state functional connectivity. JAMA Psych. 2015;72:603-611.

2. Goldberg SB, Tucker RP, Greene PA, Davidson RJ, Wampold BE, Kearney DJ, Simpson TL. Mindfulness-based interventions for psychiatric disorders: A systematic review and meta-analysis. Clin Psychol Rev. 2018;59:52-60.

3. Fox KCR, Dixon ML, Nijeboer S, Girn M, Floman JL, Lifshitz M, et al. Functional neuroanatomy of meditation: A review and meta-analysis of 78 functional neuroimaging investigations. Neurosci Biobehav Rev. 2016;65:208-228.

4. Fox KCR, Nijeboer S, Dixon ML, Floman JL, Ellamil M, Rumak SP, et al. Is meditation associated with altered brain structure? A systematic review and meta-analysis of morphometric neuroimaging in meditation practitioners. Neurosci Biobehav Rev. 2014;43:48-73.

5. Creswell JD, Taren AA, Lindsay EK, Greco CM, Gianaros PJ, Fairgrieve A, et al. Alterations in resting-state functional connectivity link mindfulness meditation with reduced interleukin-6: A randomized controlled trial. Biol Psychiatry. 2016;80:53-61.

6. Shou H, Yang Z, Satterthwaite TD, Cook PA, Bruce SE, Shinohara RT, et al. Cognitive behavioral therapy increases amygdala connectivity with the cognitive control network in both MDD and PTSD. Neuroimage Clin. 2017;14:464-470.

7. Schilbach L, Bzdok D, Timmermans B, Fox PT, Laird AR, Vogeley K, Eickhoff SB. Introspective minds: using ALE meta-analyses to study commonalities in the neural correlates of emotional processing, social \& unconstrained cognition. PLoS One. 2012;7:e30920.

8. Hasenkamp W, Barsalou LW. Effects of meditation experience on functional connectivity of distributed brain networks. Front Hum Neurosci. 2012;6:38. 
178

179 9. Ma Y. Neuropsychological mechanism underlying antidepressant effect: a systematic meta-analysis.

180 Mol Psychiatry. 2015;20:311-319.

181

182 10. Perrin JS, Merz S, Bennett DM, Currie J, Steele DJ, Reid IC, Schwarzbauer C. Electroconvulsive 183 therapy reduces frontal cortical connectivity in severe depressive disorder. Proc Natl Acad Sci USA. $184 \quad 2012 ; 109: 5464-5468$. 
Lifshitz et al. 8

185

186

187

188

189

190

191

192

193

194

195

196

197

198

199 200

\section{Figure Legends}

Figure 1. Changes in resting-state functional connectivity associated with mindfulness-based therapy for major depression. (A) Seed-based spreading interaction analysis revealed decreases in resting-state functional connectivity between the DLPFC seed and three clusters (bilateral fusiform gyrus and right angular gyrus) from pre- to post-treatment in the mindfulness-based therapy group but not in the relaxation control group (whole brain-corrected $p<.05$ ). Crosshairs mark peak voxels in bilateral fusiform gyrus (right: 140 voxels, MNI coordinates [x,y,z mm: 24, -51, -12]; left: 69 voxels, [-24, -63, $15])$ and right angular gyrus (248 voxels, [36, -78, 21]). Note that multiple statistically significant clusters may be viewable in any given single slice image. (B) Mean functional connectivity ( $z$-scores) from each of the significant clusters identified in the seed-based analysis, plotted by group and time. (C) Mindfulness-based therapy led to better clinical outcomes than did the control intervention. ANCOVA revealed that post-treatment self-report depression scores (Beck Depression Inventory-II [BDI-II]) were significantly lower in the mindfulness-based therapy group $(n=14)$ compared to the relaxation control group $(n=17)$, after controlling for pre-treatment BDI-II scores $\left(F(1,28)=22.83, p<.001, \eta^{2}=.45\right)$. 

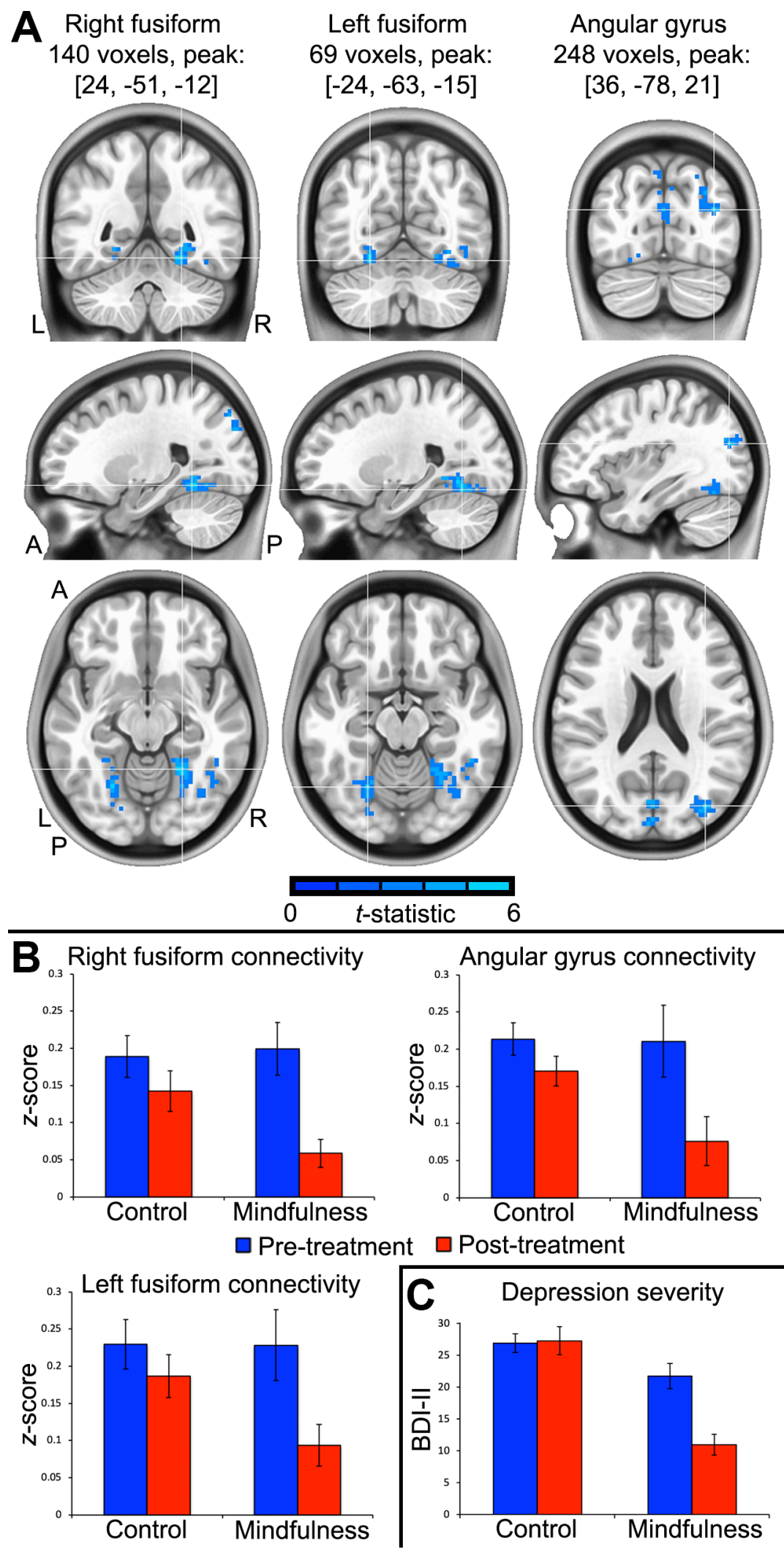


\section{Supplementary Materials}

203 For "Mindfulness-based therapy regulates brain connectivity in major depression".

\section{$\underline{\text { Participants }}$}

Participants were adult patients with chronic or recurrent MDD recruited from a larger clinical trial examining the impact of mindfulness training (ClinicalTrials.gov NCT02801513) [1]. Thirty-seven patients who had fully adhered to the treatments participated in the resting-state fMRI assessments at pre- and post-treatment, 17 of whom had been randomized to the mindfulness therapy condition and 20 to the relaxation control condition. Six of these participants were excluded due to excessive movement during the fMRI assessments (three in the mindfulness intervention, three in the control intervention), leaving a final sample of $n=14$ in the mindfulness group and $n=17$ in the control group.

Inclusion criteria at initial assessment for the trial were (a) a current diagnosis of MDD as assessed by Structured Clinical Interview for DSM-IV-TR (SCID) [2] (b) a lifetime history of depression with onset before age 19 and either chronic persistence of symptoms or a history of at least three previous episodes of depression, two of which needed to have occurred during the last two years, (c) self-reported severity of current symptoms on a clinical level as indicated by BDI-II scores above 19, (d) age 25 to 65, (e) right handedness (adopted in order to control for laterality effects), and (f) fluency in spoken and written German. Exclusion criteria were (a) history of psychosis or mania, current eating disorder, obsessive compulsive disorder, current self-harm, current substance abuse or dependence, (b) history of traumatic brain injury, and (c) current treatment with cognitive behavioral therapy. Patients who were currently taking antidepressants were allowed into the study provided that the medication had not been changed during the four weeks before entry into the study. Interviews using the Research Version of the Structured Clinical Interview for DSM-IV-TR Axis I Disorders, a well-validated semi-structured interview to determine current and past DSM-IV-TR axis-I diagnoses, were conducted by one of two trained clinical psychologists. The SCID was used to assess current and past diagnostic status at pre-treatment and current diagnostic status at post-treatment. To facilitate assessment of past episodes of depression, interviewers guided patients to construct visual timelines of depression lifetime history in order to identify episodes before assessment of criteria. Note that the time frame for the post-assessment, two weeks, partly overlapped with the intervention period. 
233 Five of the 14 participants in the final mindfulness group reported taking antidepressants at entry into the 234 study (35\%; 2 tricyclics, 3 selective serotonin reuptake inhibitors). In the control group, 5 of 17 235 participants were on antidepressants (29\%; 4 selective serotonin reuptake inhibitors, 1 selective serotonin 236 norepinephrine reuptake inhibitors), $\chi^{2}(1)=.14, p=.70$. Mean age of onset was $17.6(S D=8.3)$ years in 237 the mindfulness group and $15.0(6.6)$ years in the control group, $t(29)=.97, p=.34$. Median number of 238 previous episodes was 7.5 (range: 4, 14) in the mindfulness group and 7 (range: 3, 35) in the control 239 group, Independent Samples Median Test $p=.89$. Nine of the participants in the mindfulness group $240(64 \%)$ and 8 of the participants in the control group (47\%) suffered from comorbid anxiety disorders, $241 \chi^{2}(1)=.92, p=.33$.

243 The two groups did not differ in terms of age, gender distribution, education ( $p s>.05$, see Supplementary 244 Table 1). In addition, we found no significant differences in fMRI head motion (maximum frame 245 displacement) between the pre- and post-intervention scans in either group, or when comparing between 246 groups at either pre- or post-intervention time points ( $p \mathrm{~s}>.05$, see Supplementary Table 2).

\section{Interventions}

249 The interventions lasted two weeks, including three 1.5-hour individual sessions with trained clinical 250 psychologists (MF and EW) as well as intensive daily home practice. The three sessions followed a set 251 and manualized structure. During the first session, the therapist introduced the rationale of the treatment 252 and familiarized the participant with the main practices for the coming week. The second session started 253 with a review of experiences from the first week. The therapist addressed any questions and difficulties 254 with the practices that had arisen during the previous week, and then introduced the main practices for 255 the second week and their rationale. The third session served to debrief participants and to help them 256 establish ways of continuing the practices on their own following the end of the study should they wish 257 to do so. In addition to the individual face-to-face sessions, participants received a booklet that described 258 in detail the practices for each day along with their rationale and related psycho-educational material.

\section{Mindfulness training:}

261 Participants in the mindfulness therapy group were asked to engage in formal meditation practice for 262 about 25 minutes twice per day on all seven days of each week (14 days total) using recorded guided 
264 although they were shorter than usual in order to facilitate practice in light of the fact that patients 265 currently suffered from depression. Nonetheless, practices followed the standard MBCT sequence 266 leading from body scan meditation and mindful movement to sitting meditations focusing on the breath, 267 body sensations, sounds, thoughts, and open awareness, to practices that were more specifically focused 268 on relating to difficult experiences with acceptance and compassion. In addition to formal meditation, 269 participants were asked to engage in shorter informal practices, such as breathing spaces, that served to 270 generalize a mindful stance to activities in daily life.

\section{Relaxation control:}

273 Participants in the active control condition were asked to schedule regular rest periods as a means of 274 deliberately retreating from the activities of the day. Participants received audio files with ambient music 275 that they were free to listen to should they feel that the music might facilitate relaxation. Length and 276 frequency of the rest periods mirrored the time demands of the meditation training. Participants received 277 a plausible rationale for the control training that linked acute depression to stress and suggested rest, 278 relaxation, and disengagement from negative thinking as an initial step towards recovery.

280 Clinical outcome measure

281 Severity of depressive symptoms was assessed with the widely used self-report Beck Depression 282 Inventory-II (BDI-II) [4]. This measure consists of 21 groups of statements referring to the presence of 283 symptoms of depression over the past two weeks.

\section{Procedure}

286 The imaging study was embedded in a larger trial testing the effects of brief mindfulness training in 287 chronically depressed patients (ClinicalTrials.gov NCT02801513). Potential trial participants were 288 screened over the phone by the recruitment team for the main inclusion and exclusion criteria and those 289 likely to meet eligibility were invited to an initial assessment session during which the Structured Clinical 290 Interview for DSM-IV was conducted. Participants who met inclusion criteria continued this session to 291 fill in self-report questionnaires and to then partake in EEG assessments, the results of which have been 292 reported elsewhere [1, 5]. Self-reported severity of depressive symptoms was assessed using the BDI-II. 293 MRI assessments were conducted in a separate session within one week after the initial assessments. 294 Participation in the MRI assessments was offered as a voluntary extra to patients who took part in the 
larger trial. After the pre-treatment assessment sessions, depressed participants were randomly allocated to receive either mindfulness-based therapy or a relaxation control intervention. After the end of the intervention, participants took part in the post-treatment assessment sessions, which followed the same sequence as the pre-treatment sessions. Individuals who had been randomized into the relaxation control were offered to take part in the mindfulness-based therapy after their last assessment for the study. Randomization for the larger trial was conducted following a simple randomization protocol using a computer-generated randomization sequence (permuted blocked randomization with blocks of size 4) and sealed envelopes that remained concealed until assignment to the groups.

Participants recorded adherence to the daily practice on protocol sheets. Given the brief duration of the interventions, we defined the adequate minimum dose for the mindfulness training as having completed at least $75 \%$ of formal meditation practices. The mean rate of compliance with formal home practice was $93.8(S D=10)$ in the mindfulness group and $92.2(S D=7.1)$ in the control group.

\section{Resting-state fMRI data acquisition and preprocessing}

Following a structural scan, participants underwent an 8 min resting-state fMRI assessment, during which they were asked to rest silently while watching a white fixation cross displayed against a black background, and to remain "relaxed and awake".

Structural and functional MRI data were acquired on a Siemens Trio 3T scanner using a 12-channel radio-frequency $(\mathrm{RF})$ head coil. T1-weighted structural images were acquired with the following parameters: 176 sagittal slices covering the whole brain, repetition time $(\mathrm{TR})=1900 \mathrm{~ms}$, echo time (TE) $=2.52 \mathrm{~ms}$, flip angle $=9^{\circ}, 256 \times 256$ matrix, voxel size $1 \times 1 \times 1 \mathrm{~mm} 3$. For each resting-state measure, 257 volumes of $\mathrm{T} 2 *$-weighted echo-planar images (EPIs) were acquired with the following parameters: 37 axial slices covering the whole brain, $\mathrm{TR}=2300 \mathrm{~ms}, \mathrm{TE}=30 \mathrm{~ms}$, flip angle $=70^{\circ}, 64 \times 64$ matrix, field of view $=192 \times 192 \mathrm{~mm} 2,37$ slices, slice-timing: interleaved ascending, voxel size $=3 \times 3 \times 3 \mathrm{~mm}$.

Functional images were preprocessed using MATLAB 2012 (The Mathworks Inc., Natick, MA, USA), SPM12 (Statistical parametric mapping software, SPM; Wellcome Department of Imaging Neuroscience, London, UK; http://www.fil.ion.ucl.ac.uk), DPABI v2.1 (toolbox for Data Processing \& Analysis for Brain Imaging; http://rfmri.org/dpabi) [6] and Analysis of Functional Neuroimages (AFNI; 
326 National Institutes of Health, Bethesda, MD, USA; https://afni.nimh.nih.gov/) [7]. We reoriented 327 functional and T1 anatomical images to oblique space, then removed the first 5 functional volumes, slice 328 time corrected and realigned the functional scans, coregistered the T1 to functional data, segmented the 329 T1 using DARTEL, normalised using DARTEL, performed nuisance covariate regression using the six 330 rigid body head movement parameters and the first five principal components from white matter and 331 cerebrospinal fluid signal according to the CompCor algorithm (component based noise correction 332 method) [8]. The AFNI program 3dBlurInMask with the automask option was then used to smooth the 333 data to $4 \mathrm{~mm}$ FWHM.

During realignment, we flagged bad time points as frames with displacement exceeding $0.5 \mathrm{~mm}$ [9]. We excluded participants if their bad time point rate exceeded $15 \%$ or if any of the six rigid body head movement parameters exceeded $3 \mathrm{~mm}$ or degrees. These criteria resulted in four participant exclusions (see Supplementary Table 2).

\section{$340 \quad$ Resting-state functional connectivity analysis}

341 Functional connectivity analyses were conducted using AFNI. Brain systems of interest were identified a priori based on the depression and meditation neuroimaging literatures, and included the frontoparietal, salience, and default systems. We investigated these systems using a standard seed-based connectivity approach. Seed coordinates were selected for the placement of $10 \mathrm{~mm}$ spheres placed as followed: bilateral dorsolateral prefrontal cortex (DLPFC; MNI coordinates: -6, -50, 18), bilateral anterior insula (aINS; MNI coordinates: left -34, 22, 0; right 40, 18, 2), and bilateral posterior cingulate cortex (PCC; MNI cooridnates: left 40, 40, 36; right $-46,38,30$ ) for the frontoparietal, salience, and default networks, respectively. Coordinates were identified as the peaks of the Neurosynth reverse inference maps for "DLPFC", "anterior insula", and "posterior cingulate". Timecourses for each network were extracted as averages from network-associated seeds and correlated against every voxel in the brain, and subsequently converted to $z$-scores. We implemented a spreading interaction approach [for example, as in 10] to specifically statistically test for voxels in which the meditation group exhibited statistically significant change from pre- to post-treatment while the control group did not. Explicitly, the spreading interaction was modeled as $[-1$ (pre-treatment, meditation), -1 (pre, control), 3 (post, meditation), -1 (post, control)]. 
357 We tested three a priori hypotheses, namely, that connectivity of the (1) DLPFC, (2) aINS, and (3) PCC 358 would change as a result of meditation training. Significance of the spreading interaction maps were 359 assessed using a cluster-simulation method. Taking into account recent concerns regarding null360 hypothesis modeling [11] we used a spatial auto-correlation function for generating simulated noise 361 volumes. Noise volumes were simulated with smoothness values estimated from the smoothed data for 362 all participants. 5000 simulated datasets with bi-sided $N N=3$ thresholding indicated that $k=67$, that is, 363 that 67 or more clustered voxels with a voxel-wise $p$-value of less than $0.01(t$-stat $>2.757)$ are required 364 to reach a $p$-value of less than 0.05 corrected at the whole-brain level. $Z$-scores were subsequently extracted for each participant from identified statistically significant clusters. These values were then plotted to interpret the spreading interaction (see Figure 1B in the main manuscript). To determine brainnetwork membership when interpreting the resulting clusters, we situated identified clusters against a common 7-network functional connectivity parcellation atlas that was previously developed based on fMRI data from 1,000 individuals [12].

\section{Correlating neural and symptom measures}

372 Subsequent to our primary functional connectivity analysis, we additionally examined whether reductions in depressive symptoms were correlated with the observed mindfulness-related changes in functional connectivity. We conducted three Pearson's correlations in the mindfulness group to examine the relationship between reductions in depressive symptoms (change in BDI-II score from pre- to postintervention) and reductions in functional connectivity (change in Z-score from pre- to post-intervention) for each of the three significant clusters identified in the primary analysis (i.e., right fusiform gyrus, left fusiform gyrus, and angular gyrus). Counterintuitively, these analyses showed that, in the mindfulness group, decreases in connectivity were inversely correlated with decreases in BDI-II scores (angular gyrus: $\mathrm{r}=-.505, \mathrm{p}=.065$; right fusiform: $\mathrm{r}=-.675, \mathrm{p}=.008$; left fusiform: $\mathrm{r}=-.543, \mathrm{p}<.045$ ). That is, while reductions in connectivity with the frontoparietal control network emerged as a signature of the early effects of mindfulness meditation, such effects were more pronounced in those who had shown relatively smaller reductions in symptoms. It is possible that the observed signatures might reflect the initial effort patients use in responding mindfully to existing symptoms and that signatures might change once patients have reached a more stable state of remission. Nonetheless, it is important to note that all but the strongest of correlations are unstable at a sample size of 14 [13]. Future studies will be necessary to confirm and potentially qualify the stability and meaning of these results. 
Supplementary Materials References

1. Winnebeck W, Fissler M, Gärtner M, Chadwick P, Barnhofer T. Brief training in mindfulness meditation reduces symptoms in patients with a chronic or recurrent lifetime history of depression: A randomized controlled study. Behav Res Ther. 2017;99:124-130.

2. First MB, Spitzer RL, Gibbon M, Williams JBW. User's Guide for the Structured Clinical Interview 395 for DSM-IV Axis I Disorders SCID-I: Clinician Version. American Psychiatric Pub, 1997.

3. Teasdale JD, Williams JMG, Segal ZV. The Mindful Way Workbook: An 8-week Program to Free Yourself from Depression and Emotional Distress. Guilford Publications, 2014.

4. Beck AT, Steer RA, Brown G. Manual for the Beck Depression Inventory-II. San Antonio, TX, 401 Psychological Corporation, 1996.

5. Gärtner M, Irrmischer M, Winnebeck E, Fissler M, Huntenburg JM, Schroeter TA, et al. Aberrant 404 long-range temporal correlations in depression are attenuated after psychological treatment. Front Hum Neurosci. 2017;11:340.

6. Yan C-G, Wang X-D, Zuo X-N, Zang Y-F. DPABI: Data Processing \& Analysis for (Resting-State) 408 Brain Imaging. Neuroinformatics. 2016;14:339-351.

7. Cox RW. AFNI: software for analysis and visualization of functional magnetic resonance neuroimages. Computers and Biomedical Research. 1996;29:162-173.

8. Behzadi Y, Restom K, Liau J, Liu TT. A component based noise correction method (CompCor) for BOLD and perfusion based fMRI. Neuroimage. 2007;37:90-101. 9. Power JD, Barnes KA, Snyder AZ, Schlaggar BL, Petersen SE. Spurious but systematic correlations 417 in functional connectivity MRI networks arise from subject motion. Neuroimage. 2012;59:2142-2154. 
419 10. Creswell JD, Taren AA, Lindsay EK, Greco CM, Gianaros PJ, Fairgrieve A, et al. Alterations in 420 resting-state functional connectivity link mindfulness meditation with reduced interleukin-6: A 421 randomized controlled trial. Biol Psychiatry. 2016;80:53-61.

422

423 11. Eklund A, Nichols TE, Knutsson H. Cluster failure: Why fMRI inferences for spatial extent have 424 inflated false-positive rates. Proc Natl Acad Sci USA. 2016;113:7900-7905.

425

426 12. Yeo BTT, Krienen FM, Sepulcre J, Sabuncu MR, Lashkari D, Hollinshead M, et al. The organization 427 of the human cerebral cortex estimated by intrinsic functional connectivity. J Neurophysiol. 428 2011;106:1125-1165.

429

430 13. Schönbrodt FD, Perugini M. At what sample size do correlations stabilize?. J Res Pers. 2013;47:609$431 \quad 612$. 
433 Supplementary Table 1. Sociodemographic characteristics of depressed patients in the mindfulness434 based therapy $(n=14)$ and the relaxation control group $(n=17)$.

435

\begin{tabular}{cccc}
\hline & $\begin{array}{c}\text { Mindfulness } \\
\text { Therapy }\end{array}$ & $\begin{array}{c}\text { Relaxation } \\
\text { Control }\end{array}$ & Test \\
\hline Age & $43.4[11.3]$ & $37.3[12.0]$ & $t(29)=1.48, p=.15$ \\
(two-tailed)
\end{tabular}

436

437 Square brackets show standard deviation. 
438 Supplementary Table 2. Maximum frame displacement (movement metric) for resting-state fMRI 439 scans at each timepoint in the mindfulness-based therapy $(n=14)$ and the relaxation control group $(n=$ 440 17).

441

\begin{tabular}{cccc}
\hline & $\begin{array}{c}\text { Mindfulness-Based } \\
\text { Therapy }\end{array}$ & Relaxation Control & Test $^{1}$ \\
\hline Pre-treatment & $1.07[.42]$ & $1.00[.52]$ & $t(29)=0.41, p=.69$ \\
\hline Post-treatment & $1.05[.41]$ & $.88[.44]$ & $t(29)=1.1036, p=.28$ \\
\hline Test $^{1}$ & $t(13)=0.2, \mathrm{p}=.84$ & $t(16)=0.97, \mathrm{p}=.35$ &
\end{tabular}

442

443 Square brackets show standard deviation.

$444{ }^{1}$ T-tests were two-tailed. 